\title{
A New Reduced Rank Square Root Kalman Filter for Data Assimilation in Mathematical Models
}

\author{
Dimitri Treebushny ${ }^{1}$ and Henrik Madsen ${ }^{2}$ \\ ${ }^{1}$ Institute of Mathematical Machines and System Problems, NAS Ukraine, \\ Prospekt Glushkova, 42, Kiev, Ukraine \\ dima@env.com. ua \\ ${ }^{2}$ DHI Water and Environment, \\ Agern Allé 11, DK-2970 Hørsholm \\ hemedhi.dk
}

\begin{abstract}
The reduced rank square root filter is a special formulation of the Kalman filter for assimilation of data in large scale models that represent simple linear or complex nonlinear systems. In this formulation, the covariance matrix of the model state is expressed in a limited number of modes. In the classical implementation [15] some sort of normalization of the square-root matrix is required when variables of different scales are considered in the model. A new approach is formulated that avoids the normalization step. In addition, it provides a more cost-efficient scheme and includes a precision coefficient that can be tuned for specific applications depending on the trade-off between precision and computational load.
\end{abstract}

\section{Introduction}

The question of development, choice and implementation of suboptimal Kalman filtering [12] in engineering applications has been investigated since the first work of Kalman [6]. Suboptimal procedures save computational burden compared to a straightforward implementation of the classical Kalman filter, which may be completely infeasible in high-dimensional models. It has been shown that the Ensemble Kalman filter [3] and the reduced rank square-root filter (RRSQRT KF) [15] can be used as effective Data Assimilation procedures in modern modeling software products. In this paper the RRSRQT KF is considered.

According to the RRSQRT approach the error covariance matrix is expressed in a small number of modes, stored in a lower rank square root matrix. The number of modes is a measure of the storage and computation time required by the filter, and should be kept as low as possible. The RRSQRT algorithm includes a reduction part that reduces the number of modes if it becomes too large in order to ensure that the filter problem is feasible. However, one cannot prevent the filter from losing some covariance information during this reduction. Additionally, when considering variables of different values of magnitude as part of the state vector some sort of normalization before truncation is needed. 
Here a new and more appropriate truncation procedure is presented. According to this approach one completely avoids normalization problems, and, even more, the new truncation step needs much less computational time than the original procedure presented in [14].

The paper is organized as follows. First an overview of the classical RRSQRT KF is given. Then an additional procedure known as the Lanczos algorithm [4] is presented, followed by a short discussion and a presentation of the new TRUE RRSQRT algorithm. Finally, some tests are shown that compare the TRUE RRSQRT KF and the classical RRSQRT KF for application in the 1D radionuclide transport model RIVTOX [17].

\section{Classical RRSQRT KF}

The RRSQRT KF is based on a square-root factorization of the covariance matrix $P=S S^{T}$ where $S$ is a matrix that contains a small number $(q)$ of leading eigenvectors $s_{i}, i=1, \ldots, q$. The algorithm can be summarized as follows:

1. Forecast of the state vector

$$
x_{k}^{f}=\Phi\left(x_{k-1}^{a}, u_{k}\right)
$$

where $x_{k}$ is the state vector at time step $k, u_{k}$ is the forcing of the system, and $\Phi()$ is the model operator. Indices $f$ and $a$ denote, respectively, forecast and analysis (update). Note that a general non-linear model operator is assumed.

2. Propagation of the leading eigenvectors

$$
s_{i, k}^{f}=\frac{1}{\omega}\left[\Phi\left(x_{k-1}^{a}+\omega s_{i, k-1}^{a}, u_{k}\right)-\Phi\left(x_{k-1}^{a}, u_{k}\right)\right], i=1, . ., q
$$

where $\omega$ is a scale factor. Heemink et al. [5] argue that $\omega=1$ is a suitable choice. Smaller values of $\omega$ will increase the danger of filter divergence, whereas larger values will provide unrealistic inputs to the dynamical model.

3. Propagation of the new system noise

$$
s_{q+i, k}^{f}=\frac{1}{\omega}\left[\Phi\left(x_{k-1}^{a}, u_{k}, \omega \varepsilon_{i, k}\right)-\Phi\left(x_{k-1}^{a}, u_{k}\right)\right], \quad i=1, \ldots, l
$$

where $\varepsilon_{i, k}$ is the $i$ 'th column of the square-root of the covariance matrix of the system noise $Q_{k}$, and $l$ is the number of modes, that are used to approximate the noise covariance matrix.

4. Reduction of covariance matrix. The propagation step in (1)-(3) increases the number of columns in the error covariance matrix from $q$ to $q+l$. To reduce the number of columns, and hence keep the rank of the matrix constant throughout the simulation, a lower rank approximation of $S_{k}^{f}$ is applied by keeping only the $q$ leading eigenvectors of the error covariance matrix. The reduction is achieved by an eigenvalue decomposition of the matrix $\left(S_{k}^{f}\right)^{T} S_{k}^{f}$. If the state vector contains 
variables with different scales of magnitude, $S_{k}^{f}$ should be normalized prior to the eigenvalue decomposition.

5. Measurement update. The state vector is updated using the Kalman filter update scheme. By using a sequential updating algorithm it is not necessary to calculate the full forecast error covariance matrix, and the updating can be performed using $S_{k}^{f}$ directly. Corresponding formulae can be found in [2].

\section{Reduction Step. Problem Formulation and Discussion}

Mathematically, the reduction step can be formulated in the following form: calculate in the best possible way matrix $\bar{S}$ such that

$$
\begin{aligned}
& \bar{S} \cdot \bar{S}^{T} \cong P_{k}^{f}=S_{k}^{f} \cdot S_{k}^{f^{T}} \\
& \bar{S} \sim N^{*} q \\
& S_{k}^{f} \sim N^{*}(q+l)
\end{aligned}
$$

where

$N \quad$ the dimension of the state vector, $N>>1$,

$q$ the rank of the approximate square root matrix, $q<<N$,

$l$ the rank of the approximate square root matrix of the system noise, $l<<$.

An elegant and effective solution of the truncation problem was suggested by the author of the RRSQRT KF Martin Verlaan in his PhD thesis [14]. It is based on the well-known linear algebra fact [11]: two matrices (omitting indices) $S \cdot S^{T}$ (large) and $S^{T} \cdot S$ (small) have the same nonzero eigenvalues, in other words the same leading eigenvalues. The truncation problem (4) has then a quite straightforward solution:

$$
\begin{aligned}
& S_{k}^{f^{T}} \cdot S_{k}^{f}=L \cdot D \cdot L^{T}, \\
& \bar{S}=\left[S_{k}^{f} \cdot L\right]_{i=1, q}
\end{aligned}
$$

The first operation is a SVD decomposition of a symmetric matrix with additional non-increasing ordering of eigenvalues in a diagonal matrix

$$
D=\operatorname{diag}\left\{\lambda_{1}, \lambda_{2}, \ldots, \lambda_{q+l}\right\}, \quad \lambda_{i} \geq \lambda_{i+1}
$$

The second operation []$_{i=1, q}$ takes only the first $q$ columns of the corresponding matrix.

This solution is operational and really attractive for its computational time savings. One needs only $O\left(N *(q+l)^{2}+(q+l)^{3}\right)$ operations, which is much less then $O\left(N^{3}\right)$ in case of calculation of the full covariance matrix and its SVD decomposition $(q, l<<N)$. 
The algorithm works fine in cases where the state vector components are the values of the same physical variable. The situation becomes much worse when one has to include into the state vector two or more different physical variables of different order of magnitude. Then procedure (5) will mix all the variables together, and optimality of the truncation procedure can not be guaranteed. A simple example explains this situation.

Example: In a 1-D water flow simulation the modeled variables are water levels and discharges. They have strong interrelations so they have to be included in one state vector. The corresponding low rank approximation of the covariance matrix has a blocked structure $S=\left[\begin{array}{ll}H & Q\end{array}\right]^{T}$ where $H$ and $Q$ are low rank approximations of the covariance matrix of, correspondingly, water levels and discharges.

Usually in the case of large rivers the errors associated with water levels are of order $10^{0}(\mathrm{~m})$, while those associated with discharges could be of order $10^{1}-10^{2}\left(\mathrm{~m}^{3} \mathrm{~s}^{-1}\right)$. The structure of the two matrices $P=S S^{T}$ (large) and $\hat{P}=S^{T} S$ (small) illustrates the problem

$$
\begin{aligned}
& P=S S^{T}=\left[\begin{array}{ll}
H H^{T} & H Q^{T} \\
Q H^{T} & Q Q^{T}
\end{array}\right] \\
& \hat{P}=S^{T} S=H^{T} H+Q^{T} Q
\end{aligned}
$$

In the case of $P$ all the errors of the different variables are separated into different blocks, while for $\hat{P}$ the first item $H^{T} H$ can be even dropped because of the huge difference of orders when compared to $Q^{T} Q\left(10^{1}\right.$ and $10^{2}-10^{4}$, respectively). So, the problem of a proper weighting of the components of $S$ appears.

In [15] an extension of (5) was suggested for the case of a mixed state vector. The extended algorithm can be written in the following form:

$$
\begin{aligned}
& \hat{S}=N \cdot S_{k}^{f} \\
& \hat{S}^{T} \cdot \hat{S}=L \cdot D \cdot L^{T}, \\
& S=[\hat{S} \cdot L]_{i=1, q} \\
& \bar{S}=N^{-1} \cdot S
\end{aligned}
$$

Matrix $N$ in (7) is a normalization matrix and provides normalization of all variables to be of approximately equal order of magnitude. The question of choice of matrix $N$ is discussed in [1], [14], [15] and others. An interesting approach of choosing $N$ independently of variable units is suggested in Segers et al. [9]. Nevertheless, it has to be mentioned that there is no universal and correct procedure of choosing the normalization matrix.

Before proceeding let us remind once again the formulation of the truncation problem.

Truncation problem: Calculate in the best possible way matrix $\bar{S}$ such that

$$
\begin{aligned}
& \bar{S} \cdot \bar{S}^{T} \cong P_{k}^{f}=S_{k}^{f} \cdot S_{k}^{f^{T}} \\
& \bar{S} \sim N^{*} q \quad S_{k}^{f} \sim N^{*}(q+l)
\end{aligned}
$$


In other words the problem can be formulated as:

Calculate the small number $q$ of leading eigenpairs of the (large) matrix

$$
P_{k}^{f}=S_{k}^{f} \cdot S_{k}^{f^{T}}
$$

The following section contains a description of an algorithm that solves approximately the same problem.

\section{Lanczos Procedure}

The Lanczos algorithm first appeared in the work by Lanczos [7]. The main problem to be solved is formulated as

Find several extreme eigenpairs of a (large) real symmetric sparse matrix $A$

For the definition of objects see [4]. The algorithm itself can be characterized as an iterative procedure, and, omitting details, has the following pseudo code structure:

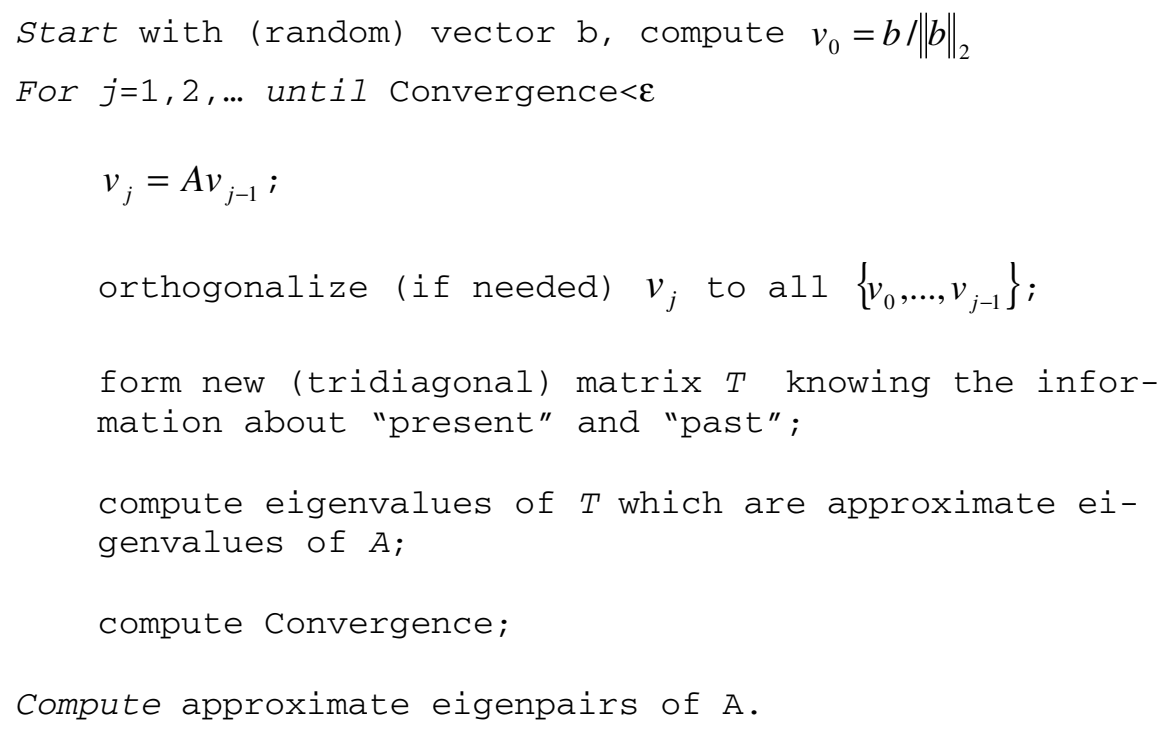

It has to be noted that one needs only several matrix-vector multiplications and some vectors operations such as scalar product during the whole algorithm. The most essential part of the procedure can be formulated as:

For the calculation of several extreme eigenpairs of a real symmetric matrix A one needs to compute several members of the sequence

$$
\left\{\begin{array}{l}
v_{0}=b /\|b\|_{2}, \\
v_{j}=A v_{j-1}, j \geq 1
\end{array}\right.
$$

with some additional vector-vector calculations. 
In the case of a large sparse matrix $A$ the operation $A v$ can be performed in a fast way using information about the sparsity. It has to be noted, however, that sparsity is not an essential part of the algorithm but an additional useful feature.

Additionally, the criterion of convergence has a specific precision parameter $\varepsilon$, so one can vary it to obtain optimized performance taking into account the trade-off between precision and computational time.

\section{The TRUE RRSQRT KF}

At this point we know how the truncation problem is formulated and, in addition, we know an effective way of solving similar problems. Before proceeding to the formulation of the new algorithm some remarks should be noted.

Remark 1: In every time step in the RRSQRT KF algorithm we have to compute the fixed (small) number $q$ of leading eigenvalues and corresponding eigenvectors of the symmetric covariance matrix $P^{*}=S^{*} \cdot S^{* T}$, where the matrix $S^{*}$ is formed in the propagation step of the RRSQRT KF filter.

Remark 2: The Lanczos algorithm provides the opportunity to compute a small number of leading eigenvalues and corresponding eigenvectors of a symmetric matrix A using several matrix-vector multiplications.

Remark 3: There is no restriction on the matrix to be sparse in order to use the Lanczos algorithm. Sparsity is used only for fast matrix-vector multiplications.

Remark 4: $P^{*} v=S^{*} \cdot S^{* T} v=S^{*} \cdot\left(S^{* T} v\right)$

Thus we can formulate the new truncation step of the TRUE (TRUncation Enhanced) RRSQRT KF algorithm:

$$
\begin{aligned}
& \text { Start with (random) vector } b \text {, compute } v_{0}=b /\|b\|_{2} \\
& \text { For } j=1,2, \ldots \text { until Convergence }<\mathcal{E} \\
& w_{j}=S^{* T} v_{j-1} ; \\
& v_{j}=S^{*} w_{j} ; \\
& \text { orthogonalize (if needed) } v_{j} \text { to all }\left\{v_{0}, \ldots, v_{j-1}\right\} ;
\end{aligned}
$$




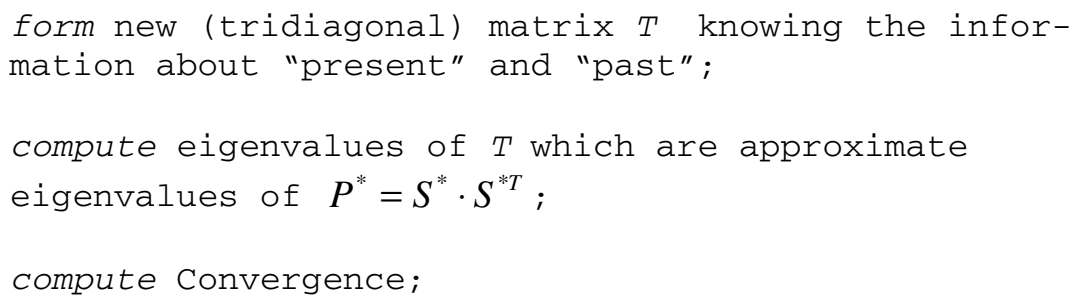

Compute approximate eigenpairs of $P^{*}$.

All other steps - propagation and update - are similar to those of the classical RRSQRT KF.

Several important features of the new truncation step should be noted:

- Normalization problems are completely avoided.

- The operations $S^{* T} v$ and $S^{*} w$ need a rather small amount of computational time because the matrix $S^{*}$ has a small number of elements. The number of iterations needed for the convergence is relatively small. Overall, the algorithm is very efficient.

- The algorithm has a specific coefficient $\varepsilon$ that regulates the precision of the algorithm. The classical RRSQRT KF is not tunable at all.

\section{Performance Tests}

Some tests for evaluating the performance of the new filter were made using the RIVTOX model. The main model equation is of an Advection-Dispersion-Reaction type. For more details see [10], [17], [18].

The purpose of the tests is to compare the precision of the approximation of the "true" covariance matrix of the state vector. The "true" covariance matrix was derived from the propagation of 1000 ensemble members using the Ensemble KF methodology [3].

Two measures of precision are defined:

1. trace $=\frac{\operatorname{Tr}\left(P_{\text {true }}-P_{\text {appr }}\right)}{\operatorname{Tr} P_{\text {true }}} \cdot 100 \%$

2. norm $2=\frac{\left\|P_{\text {true }}-P_{\text {appr }}\right\|_{2}}{\left\|P_{\text {true }}\right\|_{2}} \cdot 100 \%$

where

$\operatorname{Tr} P \quad$ is the trace of matrix $P$ - the sum of all diagonal elements,

$P_{\text {true }} \quad$ is the referred "true" system covariance matrix, computed from the statistics of 1000 ensemble members in the corresponding Ensemble KF run,

$P_{a p p r} \quad$ is the approximation of the covariance matrix, $P_{a p p r}=S \cdot S^{T}$,

$S \quad$ is the approximation of the square root of the system covariance matrix,

$\|P\|_{2} \quad$ is the second norm of matrix $P$ - the square root of the sum of squares of all matrix elements, 
For both measures a perfect approximation corresponds to trace $=$ norm $2=0$.

In the tests the main variable parameter was the number of modes $q$ for approximation of the square root matrix, and it was set to values of 5, 10, 15, 20, 25, 30 and 35 . The number of modes $l$ for approximation of the system noise covariance matrix was fixed and set equal to 10 .

For the classical RRSQRT KF the normalization according to energy suggested by Verlaan [14] was applied.

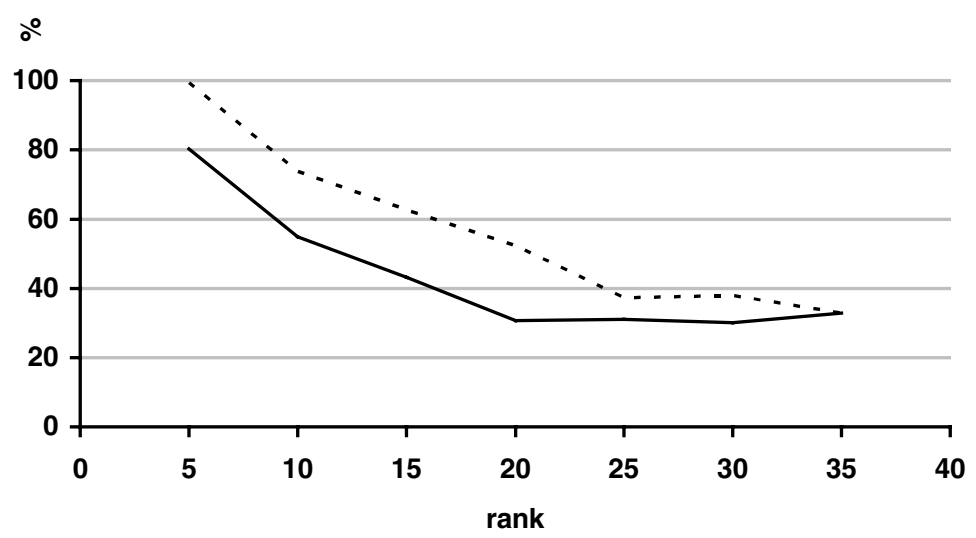

Fig. 1. Relative second norm norm2 of the error of covariance matrix approximation of the TRUE RRSQRT filter (solid line) and the classical RRSQRT filter (dotted line).

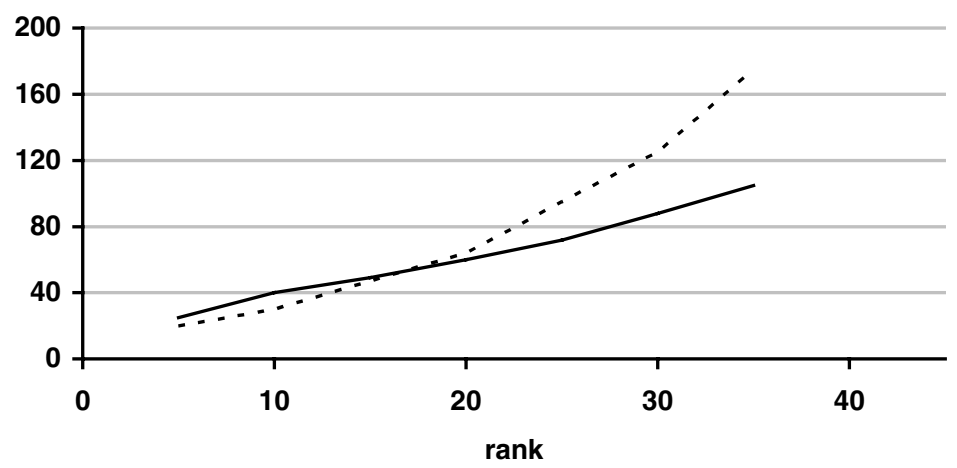

Fig. 2. Computational time [sec] of the TRUE RRSQRT filter (solid line) and the classical RRSQRT filter (dotted line).

The results of the tests are presented in Fig.1 and Fig.2. From the figures the following can be seen: 
- For the same rank the TRUE RRSQRT provides a better approximation of the covariance matrix than the classical RRSQRT. The gain in using the TRUE RRSQRT is more pronounced for smaller ranks and in the case of a zero initial covariance matrix.

- The TRUE RRSQRT is computationally more efficient than the classical RRSQRT. The classical RRSQRT requires more than twice the computational time of the TRUE RRSQRT to obtain the same level of precision of the covariance approximation. This becomes more pronounced for larger ranks since the computational time required by the classical RRSQRT has a cubic dependence on the rank and the TRUE RRSQRT has an almost linear dependence.

\section{Conclusions}

A new data assimilation algorithm has been developed - the TRUE RRSQRT (TRUncation Enhanced Reduced Rank SQuare-RooT) Kalman filter. Some tests were carried out using the mathematical model of nuclide pollution transport via 1-D river network RIVTOX to evaluate the performance of the TRUE RRSQRT filter and compare with the classical implementation of the RRSQRT. The following conclusions were obtained:

- Compared to the classical RRSQRT approach the TRUE RRSQRT filter gives a reduction of a factor 2 or more in computation time for the same level of precision of the approximation of the covariance matrix.

- The TRUE RRSQRT avoids normalization of the square-root matrix before truncation, which is a major problem with the classical RRSQRT filter when working with variables of different scales.

- The TRUE RRSQRT includes a precision coefficient that can be tuned for specific applications depending on the trade-off between precision and computational load. The classical RRSQRT filter cannot be tuned.

Acknowledgement. This work was supported by the European Commission under the contract No. FIKR-CT-2001-50021 "Data Assimilation for Off-site Nuclear Emergency Management (DAONEM-CTI)".

\section{References}

1. Cañizares, R., 1999, On the application of data assimilation in regional coastal models, $\mathrm{PhD}$ thesis, IHE Delft and TU Delft, The Netherlands.

2. Chui, C.K. and Chen, G., 1991, Kalman Filtering: With Real-Time Applications, 2nd edition. Springer-Verlag, New York

3. Evensen, G., 1994, Sequential data assimilation with a non-linear quasi-geostrophic model using Monte Carlo methods to forecast error statistics, J. Geophys. Res., 99(C5), 10,14310,162 . 
4. Golub G. and Van Loan, C, 1989, Matrix Computations, John Hopkins U. P., Baltimore.

5. Heemink, A.W., Verlaan, M. and Segers, A.J., 2000, Variance reduced ensemble Kalman filtering, Report 00-03, Department of Applied Mathematical Analysis, Delft University of Technology, The Netherlands.

6. Kalman, R.E., 1960. A new approach to linear filter and prediction theory. Journal of Basic Engineering 82D, 35-45.

7. Lanczos, C., 1950, An iterative method for the solution of the eigenvalue problem of linear differential and integral operators. J. Res. Nat. Bureau Standards, B, 45, 225-280.

8. Madsen, H. and Cañizares, R., 1999, Comparison of extended and ensemble Kalman filters for data assimilation in coastal area modeling, Int. J. Numer. Meth. Fluids, 31, 961-981.

9. Segers A.J., Heemink A.W., Verlaan M., van Loon M., 2000, A modified rrsqrt-filter for assimilating data in atmospheric chemistry models, Environmental Modeling \& Software, 15, 663-671.

10. Slavik O., Zheleznyak M., Dzuba N., Marinets A., Lyashenko G., Papush L., Shepeleva T., Mihaly B. Implementation of the decision support system for the river-reservoir network affected by releases from the Bohunice NPP, Slovakia - Radiation Protection Dosimetry, 1997, v.73, No.1-4, pp.171-175

11. Strang, G.: Linear Algebra and Its Applications. 3rd ed. International Thomson Publishing, (1988)

12. Todling, R., Cohn, S.E., 1994, Suboptimal schemes for atmospheric data assimilation based on the Kalman filter, Mon. Wea. Rev., 122, 2530-2557.

13. Van Leeuwen, J. (ed.): Computer Science Today. Recent Trends and Developments. Lecture Notes in Computer Science, Vol. 1000. Springer-Verlag, Berlin Heidelberg New York (1995)

14. Verlaan, M., 1998, Efficient Kalman filtering algorithms for hydrodynamic models, TU Delft, The Netherlands.

15. Verlaan, M., Heemink, A.W., 1995, Reduced rank square root filters for large scale data assimilation problems, Second International Symposium on Assimilation of Observations in Meteorology and Oceanography, World Meteorological Organization, 247-252.

16. Verlaan, M. and Heemink, A.W., 1997, Tidal flow forecasting using reduced rank square root filters, Stochastic Hydrol. Hydraul., 11, 349-368.

17. Zheleznyak, M, Donchytz, G., Hygynyak, V., Marinetz, A., Lyashenko, G., Tkalich, P., 1997, RIVTOX - one dimensional model for the simulation of the transport of radionuclides in a network of river channels, RODOS Report WG4-TN(97)05, Forschungszentrum Karlsruhe, 2000,48 p.

18. Zheleznyak M.J., Tkalich P.V., Lyashenko G.B., Marinets A.V. Radionuclide aquatic dispersion model-first approach to integration into the EC decision support system on a basis of Post-Chernobyl experience. - Radiation Protection Dosimetry, N6, 1993, pp.37-43. 